\title{
Dynamics of matter-wave solitons in harmonic traps with flashing optical lattices
}

\author{
T. J. Alexander, ${ }^{1, *}$ K. Heenan, ${ }^{2}$ M. Salerno, ${ }^{3}$ and E. A. Ostrovskaya ${ }^{2}$ \\ ${ }^{1}$ School of Physical, Environmental and Mathematical Sciences, University of New South Wales Canberra, Canberra ACT 2600 , Australia \\ ${ }^{2}$ Nonlinear Physics Centre, Research School of Physics and Engineering, The Australian National University, Canberra ACT 0200, Australia \\ ${ }^{3}$ Dipartimento di Fisica “E. R. Caianiello” Universitá degli Studi di Salerno, Via Ponte don Melillo, I-84084 Fisciano (SA), Italy
}

(Received 15 March 2012; published 29 June 2012)

\begin{abstract}
We study, analytically and numerically, the dynamics of a matter-wave soliton formed by a Bose-Einstein condensate with an attractive interatomic interaction confined in a one-dimensional harmonic trap and subjected to a flashing weak periodic potential. Within the framework of the Gross-Pitaevski equation, we show that efficient energy transfer to the soliton occurs within two distinct frequency regimes: (a) on resonance with the trapping frequency and (b) on resonance with the internal mode of the soliton. We find that the former regime is well described within the soliton-as-particle approximation, while the latter relies on the extended nature of the localized state and involves strong excitations of the width coupled to translational motion.
\end{abstract}

DOI: 10.1103/PhysRevA.85.063626

PACS number(s): 03.75.Lm, 03.75.Kk, 05.45.Yv

\section{INTRODUCTION}

Since their first observation [1] matter-wave bright solitons have attracted an increasing amount of interest due to their unique possibilities in both applied and fundamental problems. At the heart of many of the investigations is the challenge of matter-wave packet control and manipulation. Controlled motion is central to proposed soliton-based metrology schemes, such as in the splitting and recombination of solitonic wave packets for matter-wave interferometry $[2,3]$ or through the sampling of a local environment through quantum reflection [4]. Controlled motion is also of fundamental interest in the development of transport schemes for manipulation of Bose-Einstein condensates [5-9] and ultracold atoms [10], and more generally, for understanding the interplay of wave dynamics and external driving potentials [11-13]. However, very little is known about the behavior of solitons driven inside confining potentials, despite the fact that such systems are a natural starting point for soliton-based matter-wave metrology. In this work we determine the mechanisms of energy transfer in the simplest case of a harmonic trap, with a driving achieved through a weak flashing optical lattice, and we uncover the dependence of the soliton motion on the frequency of driving and the soliton mass.

The driving of solitons by a flashing optical lattice has been studied recently in the context of the ratchet effect $[5,6,8]$ where it was found that a one-dimensional asymmetric optical lattice can lead to directed soliton motion, however we note that regular energy exchange between the lattice and soliton leading to chaotic soliton motion was not studied in these earlier works. By contrast in this work we find that in the strong energy-exchange regime, chaotic motion becomes the norm, even in the absence of asymmetry.

In confined waves without an optical lattice a timemodulated confining potential has been shown to lead to Faraday resonances in a Bose-Einstein condensate (BEC) [14], yet the presence of a static optical lattice has been shown to suppress the Faraday wave development [15]. However the case we consider here, a static confining potential and a time-varying optical lattice, has received little attention. We use it to understand the basic mechanism of energy exchange between the lattice and a soliton and we reveal two possible pathways to soliton motion. The most robust mechanism is an effective parametric driving achieved through the coupling of the lattice to the soliton collective mode in the trap. The second mechanism for energy exchange is that of a coupling between the lattice and a soliton internal mode. We find in the absence of dissipation this ultimately leads to the break-up of the soliton, unlike in the case of sine-Gordon kink solitons where this mechanism can play a dominant role in soliton transport [16].

Our investigations are based on the mean-field GrossPitaevskii equation (GPE) and a variational approach. The latter has been successfully used to describe BEC dynamics in the absence of a time-varying potential [17]. We find that this variational method also works well in the time-dependent case, accurately predicting the qualitative behavior of the condensate. In the chaotic regime however, as expected, precise quantitative predictions of the soliton motion breakdown, although we find that the general predictions from the variational method are still valid.

Our analysis is presented as follows. In Sec. II we begin by developing the one-dimensional GPE from the full threedimensional system, discussing the limitations inherent in this approximation, and introducing the characteristic parameter values we will use throughout this work. In Sec. III we derive the variational model for our time-dependent system, obtaining a system of two coupled ordinary differential equations which accurately model the dynamics. In Sec. IV we examine the dynamics in the presence of a harmonic trapping potential, uncovering the two regimes of energy exchange with the soliton. In Sec. V we present an experimental scenario in which to observe our results, and in Sec. VI we present our conclusions.

\section{MODEL}

We begin with the full three-dimensional GrossPitaevskii equation, modeling the mean-field dynamics of the

\footnotetext{
*t.alexander@unsw.edu.au
} 
Bose-Einstein condensate:

$$
i \hbar \frac{\partial \Psi}{\partial t}=\left[-\frac{\hbar^{2}}{2 m} \nabla^{2}+V(\mathbf{r}, t)+\frac{4 \pi \hbar^{2} a_{s} N_{\text {tot }}}{m}|\Psi|^{2}\right] \Psi,
$$

where $\Psi(\mathbf{r}, t)$ is the macroscopic wave function, normalized such that $\int|\Psi(\mathbf{r}, t)|^{2} d \mathbf{r}=1, N_{\text {tot }}$ is the number of atoms, $m$ is the atomic mass, and $a_{s}$ is the $s$-wave scattering length.

Here we consider the case of a BEC with an attractive interatomic interaction $\left(a_{s}<0\right)$, trapped in the $x$ direction by a harmonic potential $V(x)$, strongly confined in the transverse $y$ and $z$ directions by a two-dimensional harmonic trap, and subject to a flashing lattice potential $U(x, t)$ along the $x$ dimension:

$$
V(\mathbf{r}, t)=V(x)+U(x, t)+\frac{1}{2} m \omega_{\perp}^{2}\left(y^{2}+z^{2}\right),
$$

where $V(x)=\frac{1}{2} m \omega_{\|}^{2} x^{2}$ is the longitudinal harmonic trap and $\omega_{\perp}$ and $\omega_{\|}$are the transverse and longitudinal harmonic trap frequencies, respectively, $U(x, t)=U_{l} E_{r} \cos \left(\omega_{l} t+\right.$ $\zeta) \cos \left(k_{l} x+\delta\right)$ is an optical lattice with wave number $k_{l}$, depth $2 U_{l}$ [in units of recoil energy $E_{r}=\hbar^{2} / \pi^{2} /\left(2 m d^{2}\right)$ where $d$ is the lattice spacing] and an intensity varying with frequency $\omega_{l}$. The parameters $\zeta$ and $\delta$ are the temporal and spatial phase offsets, respectively.

We follow the standard procedure to reduce this model to one dimension [18]. We assume the wave function to be separable, of the form $\Psi(x, y, z, t)=\psi(x, t) \phi(y, z, t)$, where $\phi(y, z, t)$ is the ground state of the two-dimensional harmonic oscillator:

$$
\phi(y, z, t)=\frac{1}{a_{\perp} \sqrt{\pi}} e^{-\left(y^{2}+z^{2}\right) / 2 a_{\perp}^{2}} e^{-i \omega_{\perp} t},
$$

with $a_{\perp}=\sqrt{\frac{\hbar}{m \omega_{\perp}}}$. Substituting the factorized wave function into the full GPE (1), multiplying by $\phi^{*}$ and integrating over the $y$ and $z$ dimensions, we derive the effective one-dimensional (1D) GPE:

$$
i \hbar \frac{\partial \psi}{\partial t}=\left[-\frac{\hbar^{2}}{2 m} \frac{\partial^{2}}{\partial x^{2}}+V(x)+U(x, t)+g_{1 D}|\psi|^{2}\right] \psi,
$$

where $g_{1 D}=2 \hbar a_{s} \omega_{\perp} N_{\text {tot }}$. This equation is valid in the weakly interacting case (i.e., when $a_{s} N_{\text {tot }}|\psi|^{2} \ll 1$ [19]).

The model is made dimensionless by the introduction of new dimensionless variables $\tilde{t}=\omega_{x} t$, where $\omega_{x}$ is a characteristic frequency in the $x$ dimension, $\tilde{x}=x / a_{x}$, where $a_{x}=$ $\sqrt{\hbar / m \omega_{x}}$, and $\tilde{\psi}=\sqrt{\frac{2\left|a_{s}\right| \omega_{\perp} N_{\text {tot }}}{\omega_{x}}} \psi$. Upon these substitutions, we arrive at the dimensionless 1D GPE:

$$
i \frac{\partial \tilde{\psi}}{\partial \tilde{t}}=\left[-\frac{1}{2} \frac{\partial^{2}}{\partial \tilde{x}^{2}}+\tilde{V}(\tilde{x})+\tilde{U}(\tilde{x}, \tilde{t})+\gamma|\tilde{\psi}|^{2}\right] \tilde{\psi},
$$

where $\gamma=\operatorname{sign}\left(a_{s}\right)$. For brevity of notation we omit the tildes in the rest of the work. The normalized potentials take the form $V(x)=\frac{1}{2} \alpha^{2} x^{2}$ for the harmonic potential, and $U(x, t)=\rho(t) \cos (k x+\delta)$ for the driving potential, where $\rho(t)=\eta \cos \left(\omega_{0} t+\zeta\right)$ (see Fig. 1). The normalized parameters in terms of the original physical parameters are given by $\alpha=$ $\omega_{\|} / \omega_{x}, \eta=U_{l} /\left(\hbar \omega_{x}\right), k=a_{x} k_{l}, \omega_{0}=\omega_{l} / \omega_{x}$. Throughout this work we set the phase offsets $\delta$ and $\zeta$ to be zero. We have found no evidence of any significant change due to variations in these phase offsets. We expect this is a consequence of the invariant time-average properties of our potential under change in these
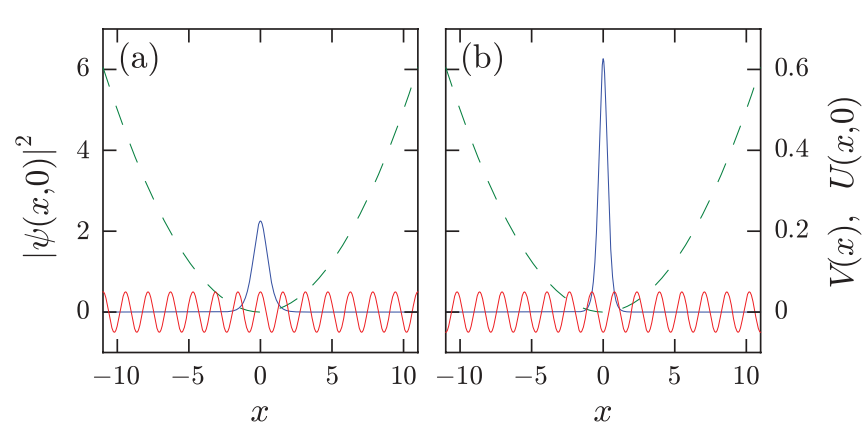

FIG. 1. (Color online) Numerical initial conditions for the soliton (dark solid line, blue) found from the variational solution within a harmonic trap (dashed line) with a flashing optical lattice (light solid line, red). (a) $N=3$ soliton, lattice at $t=0$; (b) $N=5$ soliton, lattice at $t=\pi / \omega_{0}$.

offsets, in contrast to other work where the phase offsets can lead to changes in the time average, and therefore changes in the observed behavior in the system $[5,6,20]$.

We are interested in the frequency domain which encompasses the resonant frequencies of the trapping potential and internal mode of the soliton, and length scales comparable to the width of the soliton. The strength of the harmonic potential is essentially a ratio of the trap frequency and the characteristic frequency in the $x$ dimension, $\omega_{x}$. We assume that the harmonic trap is much more extended than the characteristic spatial scale of the soliton, $a_{x}$, so that the frequency of the harmonic trap is well separated from the frequencies of the soliton internal modes. In our calculations we take $\alpha=0.1$. The total atom number $N_{\text {tot }}$ is now related to the one-dimensional soliton mass $N=\int_{-\infty}^{\infty}|\psi|^{2} d x$ by the relationship $N_{\text {tot }}=N \omega_{x} a_{x} /\left(2\left|a_{s}\right| \omega_{\perp}\right)$. Note that the strength of the nonlinearity is captured by $N$, increasing $\left|a_{s}\right|$ will increase $N$ with $N_{\text {tot }}$ fixed.

For the driving potential, we restrict our attention only to the effects of the lattice wave number $k$ and frequency of oscillation $\omega_{0}$, and fix the amplitude of the driving potential at $\eta=0.05$.

\section{VARIATIONAL ANALYSIS}

The variational approach $[17,21,22]$ allows us to reduce the complexity of the full nonlinear wave system (5) to a problem involving ordinary differential equations for the variables of interest (such as the center-of-mass position). We follow a similar procedure to that used in Ref. [17] to find a set of equations describing the dynamics of the width, position, amplitude, and phase of a soliton in the presence of a potential. In the limit of constant width (which typically occurs when the system is driven far from the soliton internal mode frequencies), the system reduces to the single equation for the soliton position.

Our derivation begins with the Lagrangian density of the GPE (5):

$$
\begin{aligned}
\mathcal{L}= & \frac{i}{2}\left(\psi \frac{\partial \psi^{\dagger}}{\partial t}-\psi^{\dagger} \frac{\partial \psi}{\partial t}\right)+\left|\frac{\partial \psi}{\partial x}\right|^{2} \\
& +[V(x)+U(x, t)]|\psi|^{2}+\frac{\gamma}{2}|\psi|^{4} .
\end{aligned}
$$


Our variational ansatz is the following sech function description of the soliton:

$\psi_{\mathrm{ans}}=A(t) \operatorname{sech}\left(\frac{x-x_{0}(t)}{w(t)}\right) e^{i\left[v(t)\left(x-x_{0}(t)\right)+\beta(t)\left(x-x_{0}(t)\right)^{2}\right]}$,

where $x_{0}, w, v, \beta$, and $A$ are, respectively, the position, width, velocity, chirp, and amplitude of the wave function. See Figs. 1(a) and 1(b) for examples of solitons of mass $N=3$ and $N=5$, respectively. Substituting this ansatz into (6) we obtain the effective Lagrangian:

$$
\begin{aligned}
L_{\mathrm{eff}}= & \int_{-\infty}^{\infty} \mathcal{L}\left[\psi_{\mathrm{ans}}\right] d x \\
= & i\left(A \dot{A}^{*}-A^{*} \dot{A}\right) w+\frac{2 \gamma}{3}|A|^{4} w \\
& +|A|^{2}\left\{-2 v w \dot{x}_{0}+\frac{\pi^{2}}{6} w^{3} \dot{\beta}+\frac{1}{3 w}+v^{2} w\right. \\
& +\frac{\pi^{2}}{3} \beta^{2} w^{3}-\alpha_{1} w\left(2 x_{0}^{2}+\frac{\pi^{2}}{6} w^{2}\right) \\
& +\alpha_{2} w\left(2 x_{0}^{4}+\pi^{2} x_{0}^{2} w^{2}+\frac{7 \pi^{4}}{120} w^{4}\right) \\
& \left.+k \pi w^{2} \eta \cos \left(\omega_{0} t\right) \cos \left(k x_{0}\right) \operatorname{csch}\left(\frac{k \pi w}{2}\right)\right\} .
\end{aligned}
$$

The Euler-Lagrange equations are given by

$$
\frac{d}{d t} \frac{\partial L_{\mathrm{eff}}}{\partial \dot{q}_{j}}=\frac{\partial L_{\mathrm{eff}}}{\partial q_{j}},
$$

where $q_{j}$ are the parameters $x_{0}, w, v, \beta, A$, and $A^{*}$. The Euler-Lagrange equation for $q_{j}=v$ gives $v=\dot{x}_{0}$ as expected. Subtracting the equations for $A$ and $A^{*}$ gives us $\frac{d}{d t}\left(w|A|^{2}\right)=0$, which after recognizing that for our soliton ansatz $N=2 w|A|^{2}$ simply means that mass is conserved in our system, also as expected. Using this result for the equation for $\beta$, we obtain $\dot{w}=$ $2 \beta w$. After some lengthy, but straightforward, algebra and using all of the equations for $A, A^{*}, x_{0}, \beta$, and $v$, we obtain the following two equations for the position and width of the soliton:

$$
\begin{aligned}
\ddot{x}_{0}= & -\alpha^{2} x_{0}+\frac{1}{2} k^{2} \pi w \eta \cos \left(\omega_{0} t\right) \sin \left(k x_{0}\right) \operatorname{csch}\left(\frac{k \pi w}{2}\right), \\
\ddot{w}= & \frac{4}{\pi^{2} w^{3}}+\frac{2 \gamma N}{\pi^{2} w^{2}}-\alpha^{2} w+3 k^{2} w \eta \cos \left(\omega_{0} t\right) \cos \left(k x_{0}\right) \\
& \times \operatorname{csch}\left(\frac{k \pi w}{2}\right)\left[\operatorname{coth}\left(\frac{k \pi w}{2}\right)-\frac{2}{k \pi w}\right] .
\end{aligned}
$$

It is worth noting that in the absence of the flashing potential there is no coupling between the soliton position and its width, and the motion is perfectly periodic. The presence of the flashing optical lattice leads to the possibility of chaotic behavior, through the introduction of nonlinearity in the soliton potential, and coupling between the collective coordinates.

\section{NUMERICAL RESULTS}

For comparison between the two methods [variational and numerical solution of Eq. (5)] we use the same initial conditions in each case: a slightly perturbed solution to the
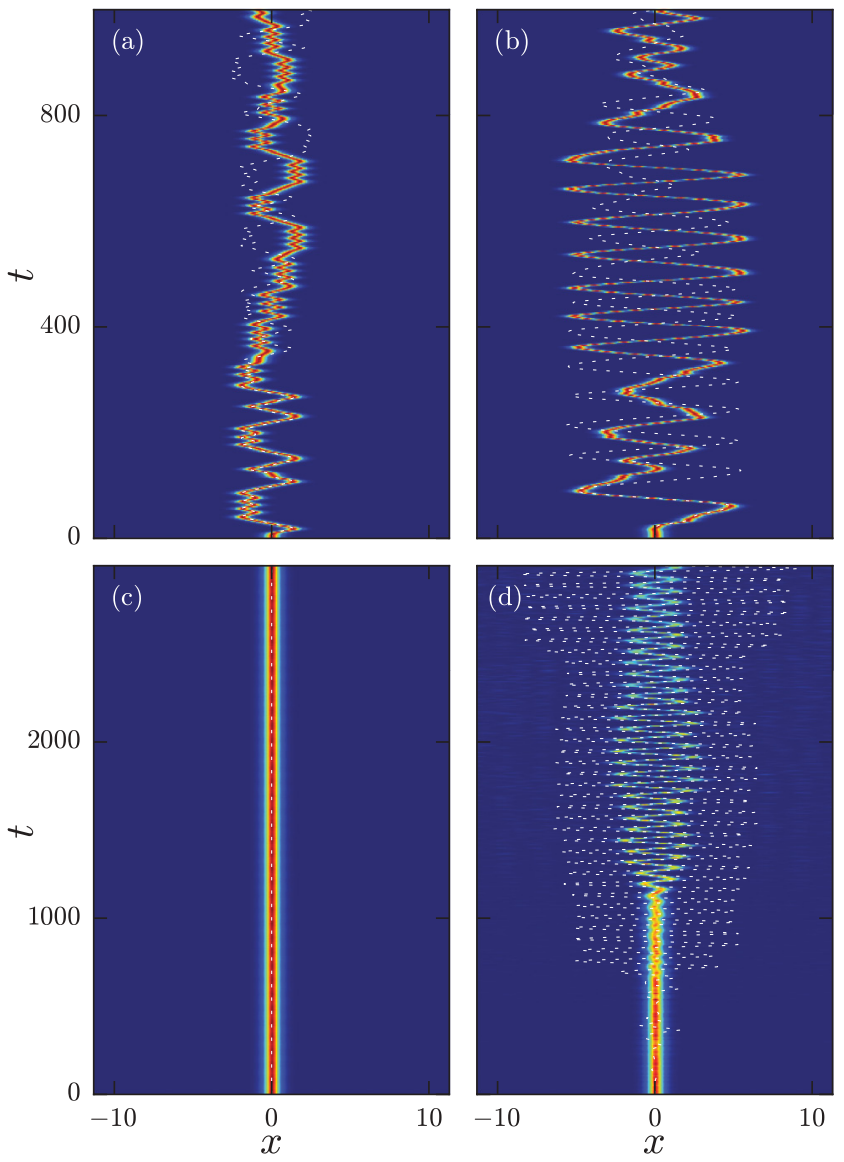

FIG. 2. (Color online) Comparison between GP (color shading of density) and variational (dotted lines) results for the four different regimes of behavior: (a) low driving frequency relative to soliton speed, $\omega_{0}=0.05$; (b) $\omega_{0}=0.5$, chaotic driving in the effectiveparticle regime; (c) $\omega_{0}=1$, no energy transfer observed; (d) $\omega_{0}=4$, excitation of the soliton width oscillation leading to center-ofmass motion. Initial conditions: $x_{0}(0)=0.001, w(0)=0.399$; other parameter values: $N=5, k=4, \eta=0.05, \alpha=0.1$.

variational equations of motion (10) and (11) with $\eta=0$ (equivalent to a small perturbation of less than $1 \%$ to the soliton from the exact soliton ground state). For the $N=5$ soliton (used for the majority of the analysis) we take $w=$ 0.399 (which gives $|A|^{2}=N / 2 w \approx 6.26$ ) and $x_{0}=0.001$ (unless otherwise noted). These initial conditions are shown in Fig. 1(a).

Numerical integration of Eq. (5) reveals four regimes of behavior for different parameter values, as identified in Fig. 2. At low driving frequencies the soliton motion is more rapid than the changing potential, and this leads to the soliton shuffling between the transient minima produced by the driving potential, with complex dynamics emerging due to the interplay between the soliton motion and the relatively slow changing potential [see Fig. 2(a)]. At a driving frequency near the trap frequency, the center-of-mass (COM) motion of the soliton is excited and we see significant spatial oscillations of the soliton [Fig. 2(b)]. The soliton itself remains almost unchanged, and as such we refer to this as the "effectiveparticle" regime. Increasing the driving frequency further we see an abrupt cessation of soliton motion [Fig. 2(c)]. Finally, at 


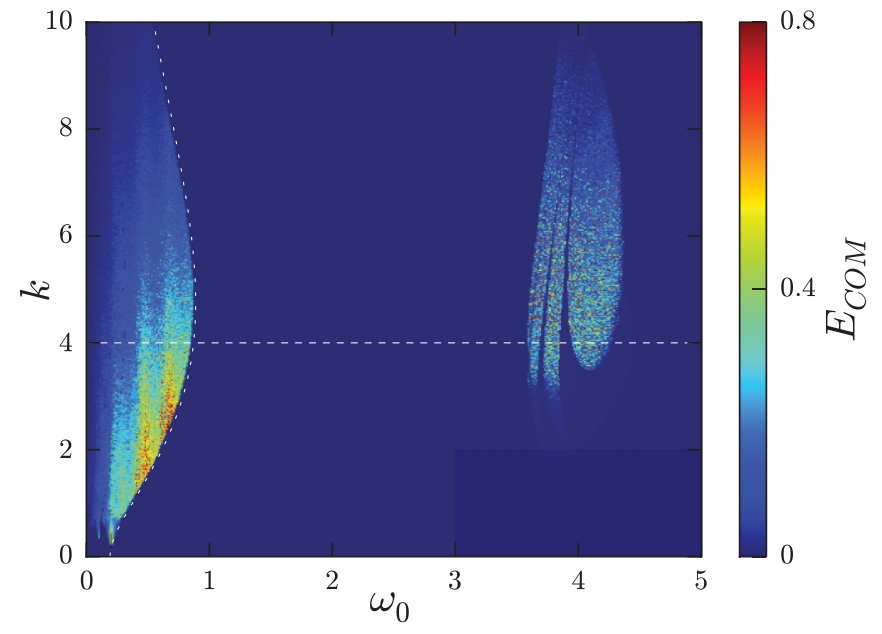

FIG. 3. (Color online) Maximum energy transfer to soliton center-of-mass $\left(E_{\mathrm{COM}}\right)$ as $k$ and $\omega_{0}$ are varied, as predicted by variational equations of motion for initial conditions $x_{0}(0)=0.001$, $w(0)=0.399$. Colors give maximum $E_{\mathrm{COM}}$ according to the color bar on the right. Existence of two distinct driving regimes can be seen, low-frequency effective-particle regime and higher frequency width oscillation regime. Parameter values are as follows: $\alpha=0.1, N=$ $5, \eta=0.05$. Dotted line corresponds to the predicted driving cutoff (see text). Dashed line corresponds to the cut at $k=4$ shown in Fig. 4.

still higher frequencies, on resonance with the internal mode of the soliton, width oscillations of the soliton are excited, which eventually couple to the center-of-mass motion [Fig. 2(d)]. As such we refer to this as the "width-oscillation" regime. The dashed white lines in Fig. 2 correspond to the solution of the variational equations of motion [Eqs. (10) and (11)]. We see that the variational approach appears to break down in the width oscillation regime [Fig. 2(d)], predicting significantly larger COM motion than observed in the GPE results. This discrepancy is due to a breakdown in the assumption that the soliton maintains its form. In fact, large shape deformations occur due to coupling to the internal modes, and thus energy is converted into deformation of the soliton rather than into COM motion. For the other cases, due to the chaotic motion of the soliton, exact agreement between the variational and numerical solutions is impossible, however the qualitative predictions of the maximum extent of the soliton motion agree well.

Given the qualitative agreement of the variational approach we now use it to understand how varying the two key driving potential parameters, the driving potential wave number $k$ and the driving frequency $\omega_{0}$, affects the transfer of energy to the soliton COM motion:

$$
\begin{aligned}
E_{\mathrm{COM}}= & \frac{1}{2} \dot{x}_{0}^{2}+\frac{1}{2} \alpha^{2} x_{0}^{2} \\
& +\frac{1}{2} k \pi w \eta \cos \left(\omega_{0} t\right) \cos \left(k x_{0}\right) \operatorname{csch}\left(\frac{k \pi w}{2}\right) .
\end{aligned}
$$

To this end we solve the variational equations of motion (10) and (11) for $k \in[0,10]$ and $\omega_{0} \in[0,5]$ and record the maximum $E_{\mathrm{COM}}$ along a single trajectory from $t=0$ to $t=3000$ with initial conditions $x_{0}(0)=0.001$ and $x_{0}^{\prime}(0)=0$. The results, shown in Fig. 3, confirm the earlier findings of Fig. 2 that there are two distinct regions of parameter space

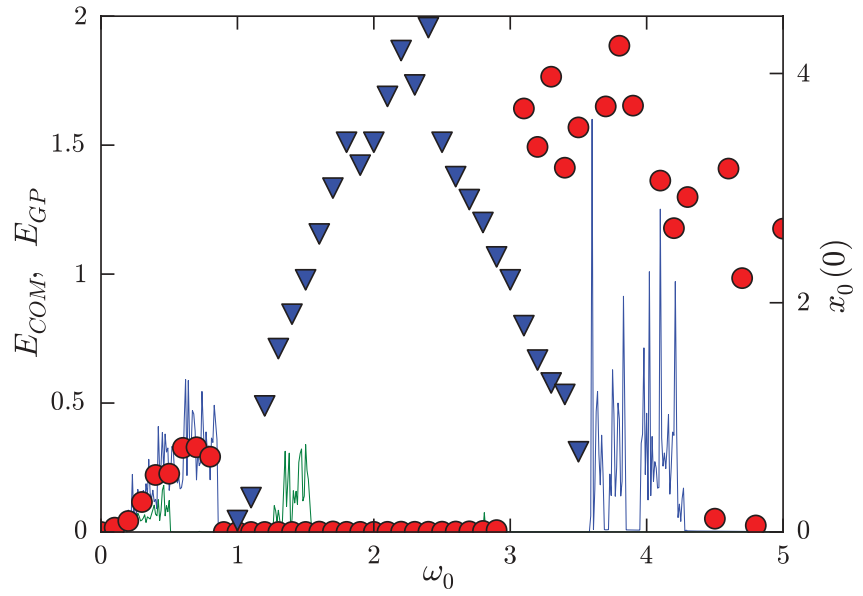

FIG. 4. (Color online) Dependence of $E_{\mathrm{COM}}$ on $\omega_{0}$ for $k=4$ and $N=5$ (blue/gray line around $\omega_{0}=0.5$ and $\omega_{0}=4$ ) and $N=3$ (low amplitude green/gray line around $\omega_{0}=0.3$ and $\omega_{0}=1.4$ ) showing shift in frequency with particle number for internal mode energy transfer, calculated using variational model and initial conditions $x(0)=0.001$ and $x^{\prime}(0)=0$. Red circles give corresponding GP results, $E_{\mathrm{GP}}$, for $N=5$. Blue triangles show the size of $x_{0}(0)$ required to initiate driving (according to right-hand axis), as predicted by the variational model $(N=5)$. Other parameters are as follows: $k=4$, $\eta=0.05, \alpha=0.1$.

for which energy is transferred to the soliton. The first region occurs near resonance with the trap's oscillator frequency of 0.1 , in the effective-particle regime. The second is the width oscillation regime, around the internal mode frequency for width oscillations; 3.99 for $N=5$. The remainder of this section seeks to uncover some of the subtleties of these results.

We begin by first examining the energy response along the dashed line in Fig. 3 corresponding to $k=4$. The results are shown in Fig. 4 as the solid line (blue). The energy transfer profile displays spikes and dips, as well as abrupt cutoffs (the extended regions where no transfer occurs). Both of these features can be traced back to the fixed initial conditions and fixed maximum time used in the calculations, and we shall discuss these effects shortly. To determine the validity of the variational approach for calculating the maximum energy transferred we compare the results with the full numerical simulation of the model (5) for the same initial conditions. Using the system Hamiltonian,

$$
H(t)=\int_{-\infty}^{\infty}\left|\frac{\partial \psi}{\partial x}\right|^{2}+(V(x)+U(x, t))|\psi|^{2}-\frac{1}{2}|\psi|^{4} d x,
$$

the energy transferred to the soliton (scaled by the soliton mass) is given by

$$
E_{\mathrm{GP}}(t)=\frac{H(t)-H(0)}{N} .
$$

The results are shown as solid circles (red) in Fig. 4 and are given by the maximum value of $E_{\mathrm{GP}}$ obtained on a single trajectory. There is good agreement between the variational approach and the full GPE results for the effective-particle regime and for the low frequency cutoff around $\omega_{0}=0.9$, however there is some discrepancy in the width oscillation regime 
around $\omega_{0}=4$. The variational approach appears to underestimate the energy transfer, and it also predicts a more limited frequency range for which the transfer occurs. As mentioned earlier, this is due to the excitation of higher-order modes in the full numerical simulation and the resulting strong deviation from the soliton ansatz. This will be discussed further below.

To explore the possible effects of different size solitons we consider briefly the case of $N=3$. The energy transfer for this soliton is shown as the light solid (green) line in Fig. 4. The most significant result is the shifting of the width oscillation regime to lower frequencies. This is exactly as expected for the internal mode mechanism. The broader $N=3$ soliton has a width oscillation frequency of approximately 1.4, which agrees well with the frequency range for which excitation occurs. This suggests that the internal mode mechanism may be useful for selectively exciting solitons based on their mass, however ultimately this excitation leads to the break-up of the soliton. As the main distinction between the different mass solitons is simply the change in width-excitation frequency, we will proceed with only the $N=5$ soliton case.

We now return to our claim that the features in the energy transfer profile of Fig. 4 come from the initial conditions (for both the effective-particle and the width oscillation regimes). The initial condition dependence is most easily visualized in the effective-particle Poincaré map, shown in Fig. 5. This map has been constructed by integrating Eq. (10), with $w=0.399$ held fixed, for a wide range of initial conditions and recording for each trajectory the values of $x_{0}$ and $x_{0}^{\prime}$ at the driving frequency. The fixing of the width is necessary to remove high energy chaotic trajectories with large width variation which can appear near the $\left(x_{0}, x_{0}^{\prime}\right)$ origin, yet are well separated in energy from our states of interest. Such states with high energy and large width oscillations are unphysical, as break-up of the soliton would occur before such excitations are observed. We may thus safely exclude such states, and so simplify our analysis of the Poincaré map. As can be seen in Fig. 5 for $\omega_{0}=0.5$ the phase space is dominated by a large chaotic region which encompasses the origin. Thus a soliton starting near the origin is already inside the chaotic layer and may explore the whole chaotic attractor (i.e., we expect the chaotic dynamics to be ergodic). The variability in maximum energy transfer is due to the limited time (up to $t=3000$ ) for which we follow the chaotic trajectory. We expect overall that a trajectory will trace out the chaotic layer over long times, however for the relatively short time examined here there can be significant variability in the maximum extent achieved by a given trajectory in the chaotic region.

The origin of the abrupt cutoff in energy transfer is evident if we consider the Poincare map at a frequency within the cutoff region, for instance at $\omega_{0}=2$ [Fig. 5(b)]. In this case we see that the origin is no longer in the chaotic layer and instead the trajectory of a soliton starting near the origin is confined to lie on a quasiperiodic orbit about the origin for which little energy is transferred. We can explore the appearance of the abrupt cutoff for different initial conditions. The triangles shown in Fig. 4 correspond to the initial position $x_{0}(0)$ required to see energy transfer to the soliton [under the condition that $x_{0}^{\prime}(0)=0$ ] and the values give a measure of the extent of the quasiperiodic orbits about the origin. As is clear in Fig. 4 there is an almost linear growth and decay of the width of
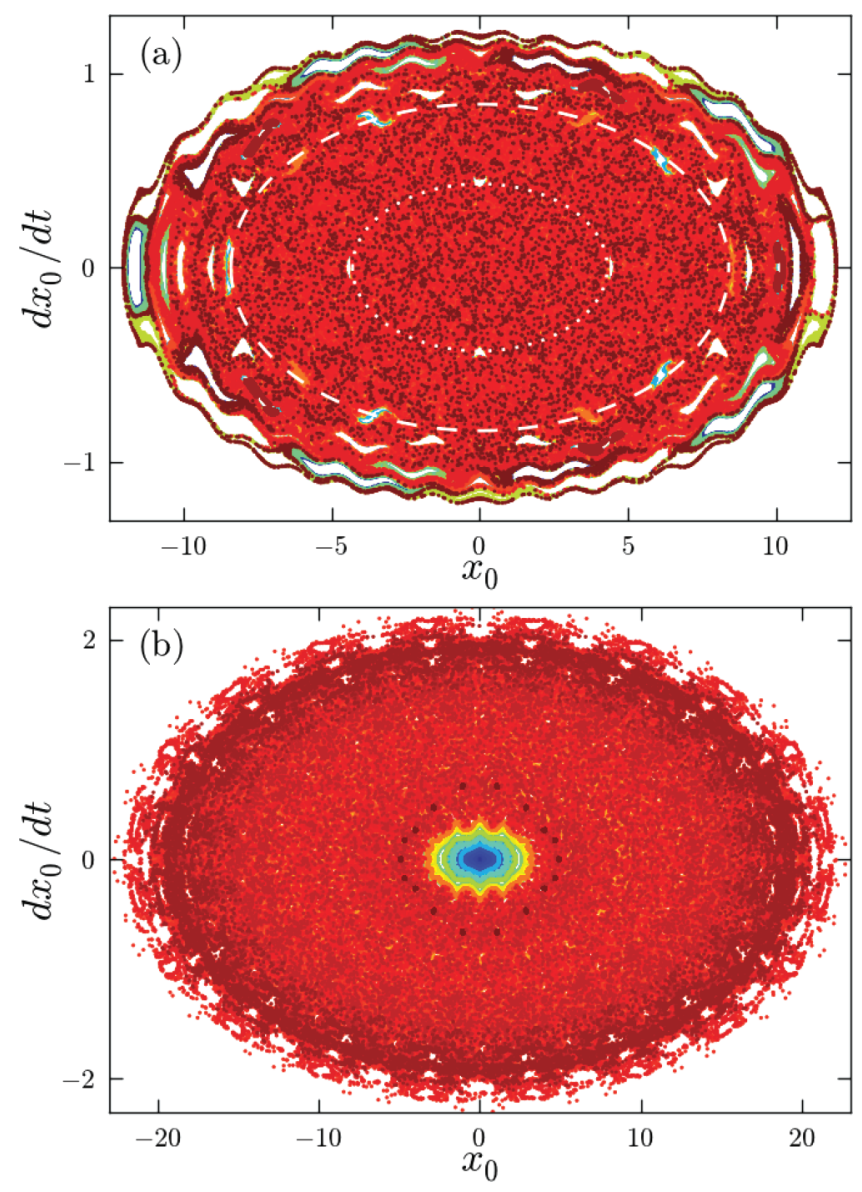

FIG. 5. (Color online) Poincaré plots for $N=5, k=4$, and (a) $\omega_{0}=0.5$ showing chaotic layer, and (b) $\omega=2$ showing existence of quasiperiodic orbits at origin. The latter leads to an absence of driving for small initial offset. Dashed and dotted lines correspond to approximate maximum energy in the chaotic layer, and lowest energy of quasiperiodic orbits, respectively. Calculation performed with variational model with $w=0.399$ fixed. Other parameters are as follows: $\eta=0.05, \alpha=0.1$.

the quasiperiodic layer about the origin. This linear growth in the perturbation required to see driving is reminiscent of parametric resonance and indeed we find the two are closely linked. The position of the bifurcation from driving to non-driving is analytically predicted by linearization of Eq. (10) near the minimum of the potential. After linearization we obtain the Mathieu equation,

$$
\ddot{x_{0}}+\left(\alpha^{2} x_{0}-A \cos \left(\omega_{0} t\right)\right) x_{0}=0,
$$

where $A=\frac{1}{2} \eta k^{3} \pi w \operatorname{csch}(k \pi w / 2)$. Standard results [23] on the stability zones of the Mathieu equation predict the parametric driving cutoff marked in Fig. 3 as a dotted line and show good agreement with our variational results. We thus see that for a soliton close to the trap center the underlying mechanism of energy transfer in the effective-particle regime is the parametric driving of the collective trap mode. In the absence of a trapping potential we see no ongoing energy transfer (although a soliton may be given kicks when the effective potential is suddenly changed as observed in Ref. [5]). In terms of the phase space of the full nonlinear system, energy transfer occurs whenever the soliton is within the chaotic layer. 

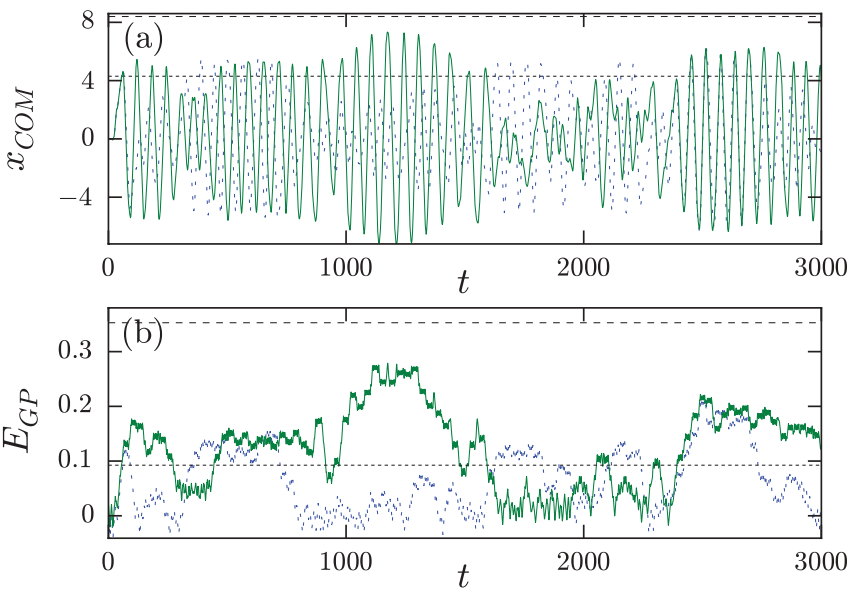

FIG. 6. (Color online) Evolution of the position of the soliton center-of-mass (a) and soliton center-of-mass energy (b) for $\omega_{0}=0.5$ for the variational results (solid lines, green) and numerical solution to the GPE (dashed lines, blue). In both cases the initial conditions are $x(0)=0.001, x^{\prime}(0)=0$. Horizontal dashed and dotted lines correspond to the dashed and dotted lines shown in Fig. 5. Other parameters are $\eta=0.05, N=5, \alpha=0.1, k=4$.

The Poincaré map may also be used to predict the maximum energy transfer possible in the system. We may extract from the map the boundary of the chaotic layer [dashed line in Fig. 5(a)] and compare this with the dynamics for a given trajectory. In Fig. 6 we plot the center-of-mass motion [Fig. 6(a)] and the energy transfer [Fig. 6(b)] for a soliton driven at $\omega_{0}=0.5$, where the solid lines (in green) are the variational results and the dashed lines (in blue) correspond to the GPE simulations. On the same figures we plot the maximum extent (long dashes) predicted by the boundary of the chaotic layer in Fig. 5(a), as well as the extent corresponding to the appearance of the first quasiperiodic orbits in the chaotic layer [light dotted line in Fig. 5(a)]. We see that the variational trajectory does appear to be bounded by the limit of the chaotic region and that it only occasionally approaches the boundary. The line corresponding to the lower energy at which quasiperiodic orbits first occur appears to be around which the soliton spends most of its time. The results corresponding to the GPE simulations appear to be similar, although for the particular trajectory shown the energy transfer appears less than that predicted by the variational approach. It should be noted that in the frequency range for which no driving from the origin is predicted the maximum extent of the chaotic layer is significantly larger than the extent at lower frequency [see Figs. 5(a) and 5(b)]. Thus, while the soliton must initially be shifted relative to the origin, once driving occurs the maximum energy transfer may be large, although as we find below, in practice a large transfer of energy to the soliton will generally lead to its destruction.

We now turn our attention to the width oscillation regime predicted in Figs. 3 and 4. Unlike the parametric driving of the collective mode of the trap this energy transfer regime occurs when the driving is resonant with the internal mode frequencies for width oscillations of the soliton. The width oscillations are subsequently coupled to the center-of-mass mode of the trapping potential through the driving, so leading to centerof-mass motion of the soliton. Coupling between internal
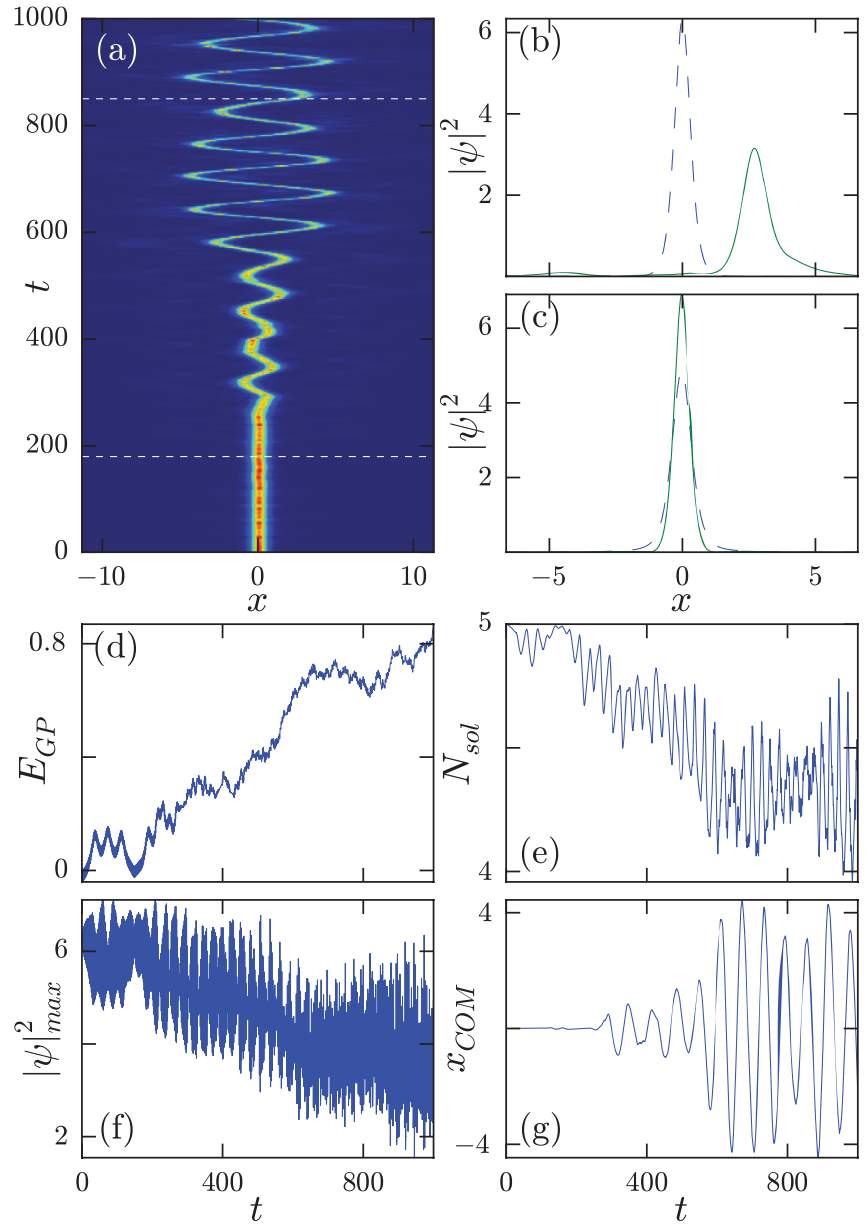

FIG. 7. (Color online) Soliton evolution according to the GPE in the width variation regime $\omega_{0}=3.1$ showing significant initial width oscillations, subsequent coupling to the collective oscillation mode, and deterioration of the soliton over time. (a) Plot of evolution with time, with colors associated with density (maximum $|\Psi|^{2} \approx 6.5$ ), dashed lines associated with density profiles on right; (b) density cut at $t=850$ showing deterioration of soliton (solid line) as compared to initial condition (dashed line); (c) plot of soliton density at $t=180$ (dashed line) and $t=181$ (solid line) showing significant width oscillations; (d) steady increase of $E_{\mathrm{GP}}$ over time through excitation of soliton internal modes; (e) decrease of soliton particle number over time due to excitations and radiation; (f) oscillation and gradual decay of soliton maximum density; (g) soliton center-of-mass position, agreeing with results in (a), and showing saturation of maximum extent.

and center-of-mass modes [16] has been seen for topological solitons ( $2 \pi$-kinks) of the sine-Gordon equation [24], however unlike in this earlier work we have no damping in our system and energy pumped into the soliton internal mode grows until the soliton is destroyed (with respect to sine-Gordon kinks our solitons are much less robust to perturbations due to their non-topological nature). We examine this response in detail in Fig. 7. In Fig. 7(a) we see the development of oscillations of the soliton position, accompanied by a decay in the maximum soliton density. Figure 7(c) shows the soliton profile at $t=180$ (dashed line) and $t=181$ (solid line) and indicates the strong width oscillations occurring in the soliton prior to center-of-mass motion. Figure 7(b) instead shows the 
soliton at $t=850$ (solid line) relative to the input profile at $t=0$ (dashed line) and the strong deviation of the soliton profile from the initial sech-type profile is clearly evident. This coupling to other soliton internal modes ultimately leads to the destruction of the soliton, and also is the origin of the poor agreement between the variational and GPE results in this regime. The steady increase of energy in the system is evident in Fig. 7(d). From approximately $t=600$ onwards the center-of-mass motion ceases to increase [the plateau is evident in Fig. 7(g)], indicating that the additional energy is being pumped into the shape deformation of the soliton (and radiation) rather than center-of-mass motion. The breakdown of the soliton is evident in Figs. 7(e) and 7(f), which show the soliton mass and soliton maximum density, respectively, with both decaying with time. We find in general that if the relative energy and mass is increased above approximately 0.5 the soliton is significantly deformed. Overall these results suggest that the width-oscillation regime may be used to selectively move solitons depending on their mass if the excitation is applied for a short time; otherwise excitation within the width-oscillation regime is an effective way to destroy a soliton in a particular mass range. We should point out that in the presence of this dynamical instability the validity of the GPE is no longer assured (see, for example, the breakdown of the GPE due to dynamical instability in Ref. [25]). We can thus only predict that destruction of the soliton will occur, but we cannot examine the detail of the breakup within the GPE theory.

\section{EXPERIMENTAL CONSIDERATIONS}

We now relate our normalized results to physical values, and provide suggestions for direct experimental verification of our work. To this end we consider the bright soliton experiment of Strecker et al. [1]. This experiment was conducted using a $\mathrm{Li}^{7}$ $\mathrm{BEC}$ and resulted in solitons with at maximum $\sim 6000$ atoms, using a scattering length of $a_{s} \approx-3 a_{0}$ (where $a_{0}$ is the Bohr radius). The radial and axial trapping frequencies in the experiment were $800 \mathrm{~Hz}$ and $70 \mathrm{~Hz}$, respectively. For our $\alpha=0.1$ this gives $\omega_{x}=\omega_{\|} / \alpha=2 \pi \times 70 / 0.1 \approx 4.4 \mathrm{kHz}$ and consequently $a_{x}=1.4 \mu \mathrm{m}$. This means that $x=1$ in our normalized units is $1.4 \mu \mathrm{m}$, and $t=3000$ is approximately $680 \mathrm{~ms}$. The physical lattice spacing corresponding to $k=4$ is then $2.3 \mu \mathrm{m}$. This relatively large lattice spacing could be achieved by intersecting the interfering lattice laser beams at an angle (see discussion in Ref. [26]). The physical lattice depth in units of recoil energy is $2 U_{l}=2 \eta \hbar \omega_{x} / E_{r}$, which for $\eta=0.05$ gives a lattice depth of $0.05 E_{r}$. The physical lattice modulation frequency corresponding to $\omega_{0}=0.5$ is $2.2 \mathrm{kHz}$, which is well within the possibilities of acousto-optic modulation [26]. Finally, $a_{s} N_{\text {tot }}\left|\psi_{\max }\right|^{2} \approx-3.4 \times 10^{-5} \ll$ 1 so the physical system is well described by the 1D GPE (5).

We find therefore that our predicted results can be observed using the standard bright soliton setup of Ref. [1] with the addition of a weak $\left(0.05 E_{r}\right)$, long-wavelength $(2.3 \mu \mathrm{m})$ optical lattice modulated at a frequency of the order of $2 \mathrm{kHz}$. For instance, to observe resonant driving of the soliton the lattice depth could be modulated at $2.2 \mathrm{kHz}$ and the system imaged after $100 \mathrm{~ms}$ to show a large spatial deviation for the soliton.
If instead the modulating frequency is increased to $5 \mathrm{kHz}$ we predict no motion will be observed, even after $500 \mathrm{~ms}$. Increasing the modulating frequency significantly further, to around $18 \mathrm{kHz}$, will lead to the destruction of the soliton by $500 \mathrm{~ms}$.

\section{CONCLUSIONS}

In this paper we have examined the energy transfer mechanisms for a soliton confined in an external potential and driven by a flashing optical lattice. In particular, we have studied the dynamics of matter-wave solitons confined in harmonic traps in the presence of time-space periodic drivings through the solution of the Gross-Pitaevskii equation, and through a variational approach which we find accurately predicts the key phenomena in the system.

We have uncovered four regimes of behavior for the driven soliton in the harmonic trap, depending on the relation between the frequencies of the soliton modes and the frequency of the driving. When the soliton motion is rapid compared to the driving potential, and the spatial scales of the soliton and driving potential are similar, we find the soliton follows chaotically the minima of the driving potential. When the driving frequency is of the order of the fundamental collective trap mode of the soliton we find that the collective mode may be excited and significant transfer of energy to the soliton center-of-mass motion may occur. We refer to this regime as the effective-particle regime since the soliton shape is only slightly perturbed during the soliton dynamics. We have shown that for small initial offsets from the trap minimum these effective-particle dynamics may be described by the Mathieu equation, indicating that the underlying mechanism for energy transfer is the parametric driving of the collective soliton mode. We have accurately predicted the frequency cutoff for this driving. At higher frequencies we have found that no energy transfer to the soliton occurs unless the soliton is initially offset from the trap minimum by an amount proportional to the detuning from the mode resonance. We have shown that ultimately energy transfer occurs because the soliton is in a chaotic layer in the phase space, and the offset is necessary to place the soliton in this layer. At higher frequencies, when the driving frequency is comparable to the width oscillation internal mode, we showed that the system enters a second regime of energy transfer. We have revealed that within this regime the driving transfers energy to the internal mode and this internal mode then couples to the collective trap mode, leading to center-of-mass motion. The internal mode frequency depends on the soliton particle number, with larger mass solitons having a higher frequency of excitation. This provides a possible energy selection mechanism based on the soliton mass. We have found, however, that prolonged excitation of the internal mode ultimately leads to the destruction of the soliton due to strong shape deformations and radiation. We suggest experimental parameters for the observation of these effects.

These results open up the possibility of understanding soliton dynamics and energy transfer in more complicated potentials and controlling the soliton dynamics through the careful tuning of a driving potential. 


\section{ACKNOWLEDGMENTS}

We thank Y. Kivshar for stimulating discussions and E.O. acknowledges useful discussions with Noel Smyth. M.S. acknowledges the Nonlinear Physics Centre and the Research School of Physics and Engineering of the Australian
National University for a visiting fellowship, and the Ministero dell' Istruzione, dell' Universitá e della Ricerca (MIUR) for partial support through a PRIN-2008 initiative. This project was partially supported by the Australian Research Council.
[1] K. E. Strecker, G. B. Partridge, A. G. Truscott, and R. G. Hulet, Nature (London) 417, 150 (2002).

[2] N. Veretenov, Y. Rozhdestvensky, N. Rosanov, V. Smirnov, and S. Fedorov, Eur. Phys. J. D 42, 455 (2007).

[3] T. P. Billam, S. L. Cornish, and S. A. Gardiner, Phys. Rev. A 83, 041602 (2011).

[4] S. Cornish et al., Physica D 238, 1299 (2009).

[5] D. Poletti, T. J. Alexander, E. A. Ostrovskaya, B. Li, and Y. S. Kivshar, Phys. Rev. Lett. 101, 150403 (2008).

[6] D. Poletti, E. A. Ostrovskaya, T. J. Alexander, B. Li, and Y. S. Kivshar, Physica D: Nonlinear Phenomena 238, 1338 (2009).

[7] J. Abdullaev, D. Poletti, E. A. Ostrovskaya, and Y. S. Kivshar, Phys. Rev. Lett. 105, 090401 (2010).

[8] M. Rietmann, R. Carretero-González, and R. Chacón, Phys. Rev. A 83, 053617 (2011).

[9] C. E. Creffield and F. Sols, Phys. Rev. A 84, 023630 (2011).

[10] Q. Thommen, J. C. Garreau, and V. Zehnl'e, Phys. Rev. A 84, 043403 (2011).

[11] A. R. Kolovsky, Phys. Rev. A 82, 011601 (2010).

[12] P. Papagiannis, Y. Kominis, and K. Hizanidis, Phys. Rev. A 84, 013820 (2011)
[13] A. Mohamadou, E. Wamba, S. Y. Doka, T. B. Ekogo, and T. C. Kofane, Phys. Rev. A 84, 023602 (2011).

[14] P. Engels, C. Atherton, and M. A. Hoefer, Phys. Rev. Lett. 98, 095301 (2007).

[15] P. Capuzzi, M. Gattobigio, and P. Vignolo, Phys. Rev. A 83, 013603 (2011).

[16] M. Salerno and N. R. Quintero, Phys. Rev. E 65, 025602 (2002).

[17] N. H. Berry and J. N. Kutz, Phys. Rev. E 75, 036214 (2007).

[18] V. M. Pérez-García, H. Michinel, and H. Herrero, Phys. Rev. A 57, 3837 (1998).

[19] L. Salasnich, A. Parola, and L. Reatto, Phys. Rev. A 65, 043614 (2002).

[20] E. Haller, R. Hart, M. J. Mark, J. G. Danzl, L. Reichsöllner, and H.-C. Nägerl, Phys. Rev. Lett. 104, 200403 (2010).

[21] D. Anderson, Phys. Rev. A 27, 3135 (1983).

[22] R. Scharf and A. R. Bishop, Phys. Rev. E 47, 1375 (1993).

[23] N. W. McLachlan, Theory and Application of Mathieu Functions (Clarendon Press, Oxford, 1951).

[24] M. Salerno and Y. Zolotaryuk, Phys. Rev. E 65, 056603 (2002).

[25] A. J. Ferris, M. J. Davis, R. W. Geursen, P. B. Blakie, and A. C. Wilson, Phys. Rev. A 77, 012712 (2008).

[26] O. Morsch and M. Oberthaler, Rev. Mod. Phys. 78, 179 (2006). 